\title{
Recurrent Schwannoma of the Ulnar nerve. Report of a case
}

\begin{abstract}
A case of a recurrent Schwannoma in the Ulnar nerve is reported, a rare type of a soft tissue benign tumour that recurs along the nerve in spite of complete removal. A more "radical" solution of that problem is discussed.
\end{abstract}

Volume 9 Issue I - 2019

\author{
Harry Kourtopoulos \\ Professor of Neurosurgery, Em latrikon Clinic of P. Faliron, \\ Greece
}

\begin{abstract}
Correspondence: Harry Kourtopoulos MD, PhD, Professor of Neurosurgery, Em latrikon Clinic of P. Faliron, Athens, Greece, Emailhkourtopoulos@yahoo.com
\end{abstract}

Received: April 28, 2018 | Published: January 24, 2019

\section{Introduction}

Schwannomas are the commonest benign tumours detected in the peripheral nerves representing approximately $8 \%$ of all soft tissue neoplasms. ${ }^{1}$ There are non-invasive, arise from the peripheral nerve sheath, encapsulated by epineurium. ${ }^{2}$ There is no sex or racial difference but there exist a 2:1 occurrence in favour of upper limbs comparing to lower while mostly develop during the $3 \mathrm{~d}$ and 6 th decade of life. ${ }^{1}$ They are slow growing, usually misdiagnosed as lipomas until they become painful. Gadolinium enhanced MRI provide a picture of an encapsulated tumour but the final picture is accomplished by exploration, removal and histopathology report.

\section{Case report}

Our present patient, a lady of now 59 years of age, was initially treated in the USA, twice, during the previous decade at age of 46 and 48 years, for a recurrent tumour of the dominant right ulnar nerve, close to the wrist. In both occasions there were, according to her description, solitary independent tumours of the volar forearm. During 2010 she noticed a new tumour, progressively painful and moderate incapacitating her movements of the right hand, hindering her to perform daily living activities. One could palpate a firm mass, perpendicularly mobile and painful. She had a positive Tinel's sign radiating along the Ulnar nerve distribution. MRI showed a 4,5x 3,0x $4,2 \mathrm{~cm}$ mass displacing the palmaris longus muscle. Gadolinium injection showed an intense enhancement (Figure 1-3).

She was taken to the theatre and the tumour was removed through a longitudinal incision and careful nerve and tumour separation. Biopsy showed a hypo-cellular tissue with Schwan cells with Antoni $\mathrm{A}$ as well as B patterns. There was no mitotic activity. The removal was total. Following the surgery, the patient had no pain and full ulnar sensory and motor activity but still some persistent numbness present.

I informed the patient for the benign nature of the tumour but also informed her of my previous experience of such tumours that tend to recur occasionally centrally, towards the brachial plexus. In those cases with frequent recurrences I had advised a radical section of the nerve together with the tumour. Of course the patient denied.

By the end of year 2015, November, the patient contacted me for a new lump. Since "some" time, meaning months, she felt a new, mobile tumour close to the right elbow. This was confirmed again by an MRI enhanced with gadolinium. There was a double mass right at the elbow $4 \times 3,5 \times 2,2 \mathrm{~cm}$ and beside, two tumour like masses of maximum $1 \mathrm{~cm}$ between the phalanges IV-III. However the patient had no complain from that region. An exploration was performed again (see photo from surgery) and complete removal was done. Biopsy showed again a bifasic neoplasm with multicellular Antoni A regions and myxoid hypo-cellular Antoni B regions. The cells were mixed with collagen fibres. There were no Verocay bodies, no mitotic activity and the resection was total. In the hand, among the phalanges, exploration was negative and wound was closed. The healing of the skin was prolonged and the numbness of the hand corresponding to the ulnar region was more prominent.

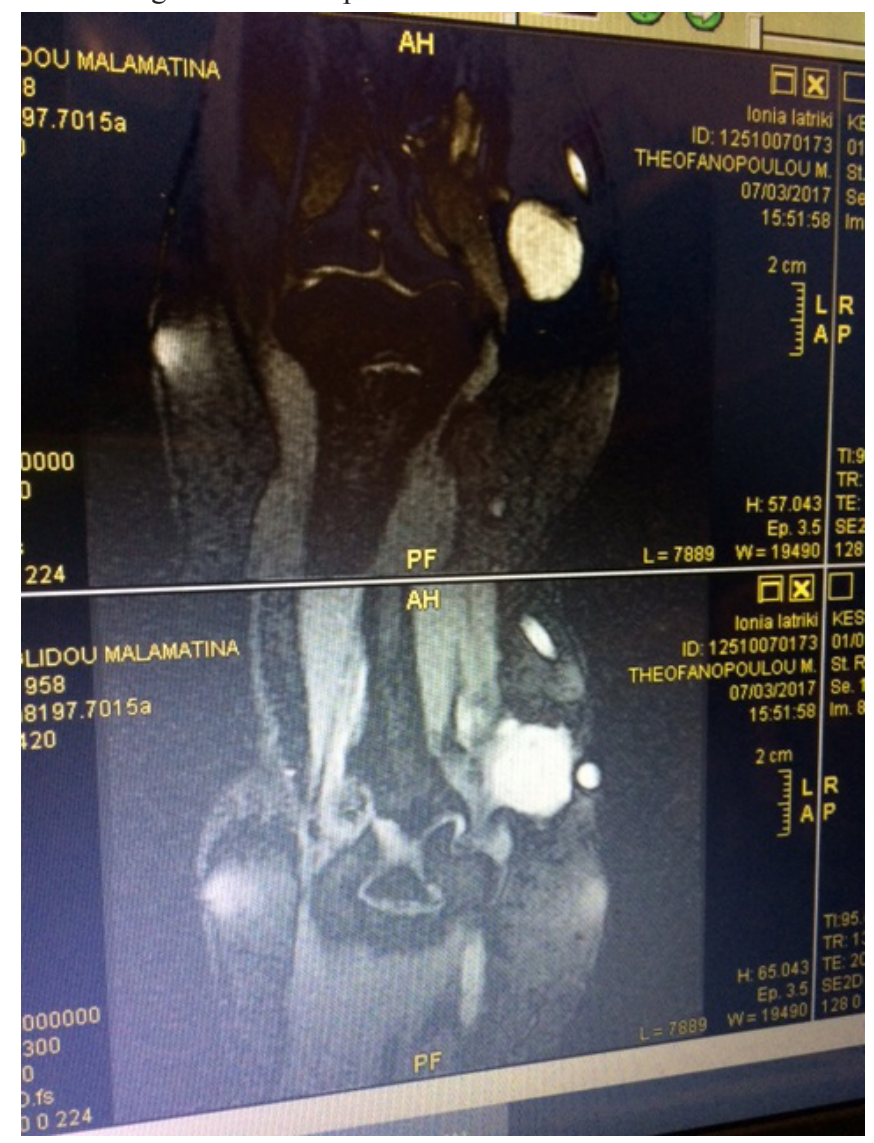

Figure 1 MRI rt elbow. 


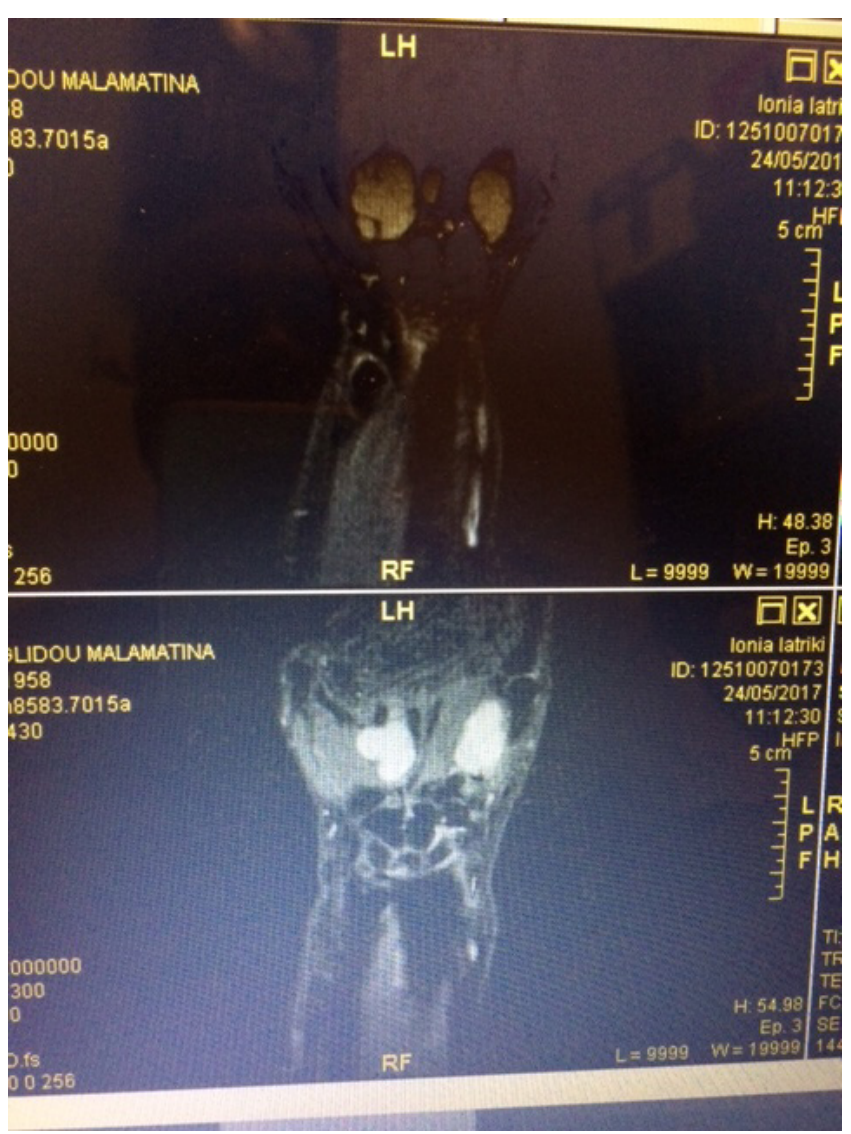

Figure 2 MRI rt wrist.

\section{Discussion}

Early diagnosis is crucial before these tumours become oversized and can create surgical problems. The symptoms appear to be nonspecific which helps patients delay and sometimes doctor's delay. Since weakness may occur if the benign tumour exceeds $2,5 \mathrm{~cm}$ in diameter, depending of the region, ${ }^{3}$ and is mobile, is easy to assume that the finding is benign and there is no rush to remove it. The same applies to other benign tumours like neurofibromas, lipomas, xanthomas and ganglion cysts. Tinel's sign is often positive and gadolinium enhanced MRI is particularly helpful. Fine needle biopsy can be rather inconclusive but certainly gives an advantage if the differential diagnosis is a ganglion cyst, but overall results are just over than $50 \%$ sufficient for diagnosis. ${ }^{4}$

Histological examination will reveal the nature of the tumour since Schwannomas are encapsulated, eccentrically located and non invasive contrary to the neurofibromas and can be removed totally. But why do they recur, in either identical locations or elsewhere along the nerve? Especially in cephalic directions towards the brachial plexus and further on? Since I have a negative experience from some cases in the past (from both ulnar and femoral nerve) I do think, as in the present case, that a more radical decision must be considered, a decision that only can be made in agreement with the patient himself.

\section{Conclusion}

Schwannomas (neurilemmomas) are rare peripheral nerve tumours that grow slowly. Early removal is advised with caution to

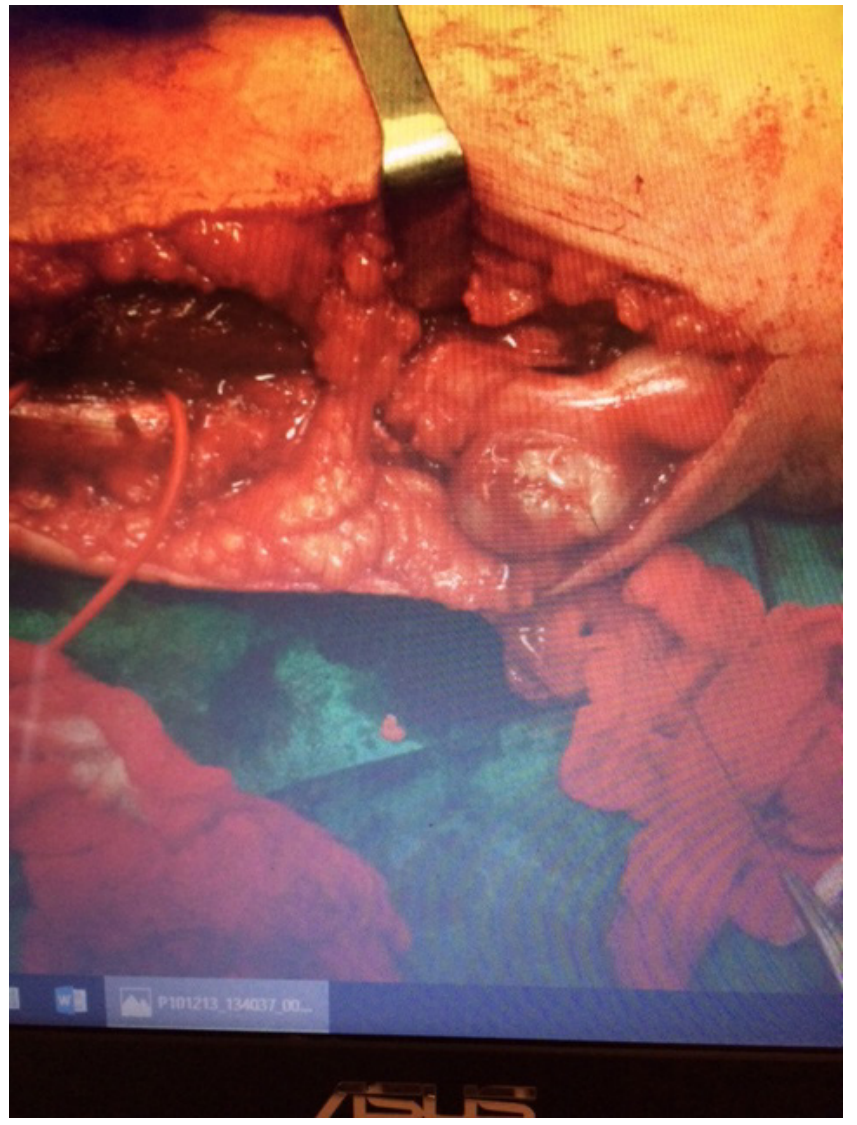

Figure 3 Operative picture of the tumour.

avoid damage to the nerve. Although recurrence is reported low, a more "radical" decision i.e. section of the nerve, can be considered in frequent-repeated re-occurrence. That decision can be made together with the patient himself.

\section{Acknowledgments}

None.

\section{Conflicts of interest}

The author declares no conflicts of interest.

\section{References}

1. Ozdemir O, Ozsoy MH, Kurt C, et al. Schwannomas of the hand and wrist: log-term results and review of the literature. J Orthop Surg (Hong Kong). 2005;13(3):267-272.

2. Lin J, Martel W. Cross-sectional imaging of peripheral nerve sheath tumours: characteristic signs on CT, MR imaging and sonography. Amer J Roentgenology. 2001;176(1):75-82.

3. Ogose A, Hotta T, Morita T, et al. Tumours of peripheral nerves: correlation of symptoms, clinical signs, imaging features and histological diagnosis. Skeletal Radiol. 1999;28:183-188.

4. Domanski HA, Akerman M, Engellau J, et al. Fine needle Aspiration of Neurilemmoma (Schwannoma). A clinicocytopathologic study of 116 patients. Diagn Cytopathol. 2006;34(6):403-412. 